\title{
Madres usuarias de servicios de preservación familiar: perfil sociodemográfico y evolución
}

\author{
Mothers as Clients of Family Preservation Services: \\ A Socio-demographic and Development Profile
}

\author{
Susana Menéndez Álvarez-Dardet \\ Universidad de Huelva \\ menendez@uhu.es \\ Ángela Victoria Arenas Rojas \\ Universidad de Sevilla \\ arenas@us.es \\ Javier Pérez Padilla \\ Universidad de Huelva \\ javier.perez@dpee.uhu.es \\ Bárbara Lorence Lara \\ Universidad de Huelva \\ barbara.lorence@dpee.uhu.es
}

Recibido: $13 / 10 / 2011$

Revisado: 28/11/2011

Aceptado: 12/12/2011

Disponible on line: 15/02/2012

\section{Resumen}

Las familias en situación de riesgo constituyen un colectivo central dentro de la población usuaria de los Servicios Sociales y, en los últimos años, se están realizando en nuestro país diversos estudios sobre ellas. En este trabajo se presentan algunos de los resultados de una investigación realizada con una muestra de madres que reciben intervenciones de preservación familiar por parte de los Servicios Sociales comunitarios de la Diputación de Huelva. En concreto se analizan sus principales características sociodemográficas y económicas, su nivel de pobreza y su historial y situación actual en los Servicios Sociales, explorando las evaluaciones que los profesionales hacen acerca de la evolución de estas familias. Asimismo, se examinan las diferencias entre las dimensiones analizadas según el tipo de servicio que trabaja con estas familias.

Palabras clave: Familias en riesgo, Servicios Sociales Comunitarios, preservación familiar, necesidades y recursos.

\begin{abstract}
At-risk families are a central group within the Social and Community Services client population and, in recent years, there is ongoing research in our country about them. In this article, we present results obtained from a survey of a sample of mothers who receive family preservation services from Social and Community Services of the Province of Huelva. Specifically, their socio-demographic and economic profile, their degree of poverty in relation to the official poverty line and their case history and current situation in Social Services while exploring the professional assessments made regarding the evolution of these families are presented. Differences in the areas analyzed according to the type of service are also examined.
\end{abstract}

Key words: At-risk families, Social and community services, Family preservation, Needs and resources.

Referencia normalizada: Menéndez Álvarez-Dardet, S., Arenas Rojas, A. V., Pérez Padilla, J. \& Lorence Lara, B. (2012): «Madres usuarias de servicios de preservación familiar: perfil sociodemográfico y evolución». Cuadernos de Trabajo Social, 25(1): 193-203.

Sumario: Introducción. 1. Método. 2. Resultados. 3. Conclusiones. 4. Referencias bibliográficas. 


\section{Introducción}

La investigación de las últimas décadas viene demostrando que, a pesar de los cambios que está experimentando en sociedades desarrolladas (Rodríguez y Menéndez, 2003), la familia sigue siendo el contexto más apropiado para dar respuesta a las necesidades evolutivo-educativas de los menores y que, por lo tanto, constituye el entorno idóneo para el crecimiento y el desarrollo infantil (López, 2008; Palacios y Rodrigo, 1998). Desgraciadamente no todas las familias pueden cumplir adecuadamente estas funciones, de manera que algunas de ellas llegan, por diversas razones, a suponer un entorno que amenaza el bienestar de niños y niñas, o bien ponen en peligro su integridad. En este sentido, la investigación contemporánea también pone de manifiesto que estas situaciones no constituyen un grupo único sino que, por el contrario, abarcan un amplio abanico de familias caracterizadas por circunstancias muy diversas que, en su conjunto, convierten en muy difícil o en imposible el adecuado desarrollo de las funciones parentales (Hidalgo, Menéndez, Sánchez, Lorence y Jiménez, 2009; Rodrigo, Máiquez, Martín y Byrne, 2008).

En un extremo de este abanico se encuentran los contextos familiares con un funcionamiento más inapropiado, en los que los menores son víctimas de diversas modalidades de malos tratos, y ante los cuales la Administración competente establece medidas de desamparo que implican separar al menor de su familia de origen y brindarle un entorno alternativo. Se trata de las situaciones más dramáticas y también las que han recibido, tradicionalmente, la atención de la Administración, aunque ni son las más frecuentes ni las únicas en las que tiene sentido intervenir. Así, y al igual que ha sucedido en otros países desarrollados, una buena parte de nuestra evolución como sociedad cada vez más moderna y progresista en materia de atención a la infancia viene definida por la incorporación de las situaciones de riesgo como ámbito de intervención de los sistemas públicos de protección de menores (De Paúl, 2009; Hidalgo, Menéndez et al., 2009). De acuerdo con Rodrigo y colaboradores (2008), las familias en riesgo psicosocial son:

Aquellas en las que los responsables del cuidado, atención y educación del menor, por cir- cunstancias personales y relacionales, así como por influencias adversas de su entorno, hacen dejación de sus funciones parentales o hacen un uso inadecuado de las mismas, comprometiendo o perjudicando el desarrollo personal y social del menor, pero sin alcanzar la gravedad que justifique una medida de amparo, en cuyo caso se consideraría pertinente la separación del menor de su familia (p. 42).

Se trata por tanto de familias en las que existe un grado variable pero importante de riesgo para el bienestar de niños y niñas, aunque la situación no alcanza tal gravedad que haga necesario declarar el desamparo $\mathrm{y}$, por tanto, separar al menor de sus progenitores. Éstos afrontan circunstancias personales y relacionales que dificultan la tarea de desempeñar adecuadamente su labor como madres y padres, pero la intervención en estas situaciones puede mejorar la atención que se brinda en la familia de origen a las necesidades del menor, sin necesidad de adoptar medidas más drásticas y, sobre todo, antes de que dichas medidas sean realmente necesarias porque la situación se haya agravado y sea pertinente apelar al desamparo. Se trata por tanto de un enfoque de trabajo capacitador, positivo, re-educativo y preventivo (Hidalgo et al., 2009; Máiquez y Capote, 2001; Rodrigo et al., 2008) que, como veremos a continuación, responde a las recomendaciones de diversos tratados internacionales y tiene reconocimiento formal en la legislación española. Según los cálculos de Rodrigo y colaboradores (2008), este tipo de familias constituyen el 80 por ciento aproximado de los expedientes que manejan los Servicios Sociales de las corporaciones locales.

La creciente visibilidad de las familias en situación de riesgo psicosocial, no sólo como espacio de intervención sino también como ámbito de estudio, ha propiciado el desarrollo de investigaciones que, desde una perspectiva sistémica y ecológica y a partir de propuestas explicativas basadas en modelos de riesgo y protección, han permitido conocer cada vez mejor este tipo de situaciones familiares. La comprensión más completa y elaborada de estos escenarios familiares, unida a los profundos cambios legales de las últimas décadas han permitido optimizar las intervenciones desarrolladas desde las instituciones públicas para 
reducir la adversidad a la que se enfrentan estas familias (Hidalgo et al., 2009).

Con toda probabilidad, una de las señas distintivas de la evolución, que ha tenido lugar en la última mitad del siglo XX en la legislación y las directrices de intervención, es el modo en que se ha ido definiendo gradualmente un sistema de protección que pasa de centrarse exclusivamente en la infancia a incorporar a la familia como objeto intervención (De Paúl, 2009; Rodrigo et al., 2008). En esta línea, las recomendaciones del Consejo de Europa (2006) para el fomento de la parentalidad positiva destacan la necesidad de conjugar acciones integradas y coordinadas entre todos los sectores sociales (con los Servicios Sociales como ámbito relevante), con objeto de mejorar la calidad de la convivencia familiar, la protección de los derechos de los menores, y contribuir así a un futuro próspero de la sociedad. Este nuevo planteamiento marca un antes y un después en la intervención con familias en situación de riesgo psicosocial, que Rodrigo y colaboradores (2008) traducen en un abordaje desde la perspectiva de la preservación y fortalecimiento familiar.

En el ámbito de la protección a la familia y a la infancia en España, los Servicios Sociales tienen como cometido la salvaguarda de los derechos de los menores y, de manera especial, asegurar que reciban la adecuada educación y protección por parte de sus cuidadores o responsables legales.

La evolución histórica en política de intervención familiar en este país (bien sintetizada en De Paúl, 2009), se inicia con cierto retraso respecto a otros países, pero ha sido crucial para las funciones que actualmente asume el sistema público de protección social. En España los Servicios Sociales fueron configurados como tales en los años 1980. La entrada en vigor de la legislación de protección infantil de 1987 dotó a las comunidades autónomas de la competencia exclusiva en materia de Servicios Sociales, y estableció un sistema de atención a familias que, como ya hemos destacado, no se centra exclusivamente en casos de alto riesgo, sino que incorpora cualquier tipo de familia que requiera ayuda profesional.

El cambio más relevante tiene lugar, sin duda, a mediados de los años noventa, cuando la Ley Orgánica de Protección Jurídica del Me- nor (Ley 1/1996) asigna a los poderes públicos la obligación de asegurar la protección social, económica y jurídica de la familia, y dentro de ésta, con carácter singular, la de los menores. En esta ley se distinguen los dos tipos de situaciones de adversidad a las que ya hemos hecho referencia: la protección ante el desamparo, que depende directamente de la Administración autonómica y que conlleva mayoritariamente medidas de acogimiento y adopción, y ante las situaciones de riesgo, que hacen posible una detección más temprana de los casos y una intervención más prolongada que la de la inminente separación (Rodríguez, Camacho, Rodrigo, Martín, y Máiquez, 2006).

Como se ha señalado, la incorporación de familias en situación de riesgo a los Servicios Sociales ha conllevado un cambio sustantivo en los modelos de intervención. En este sentido, la legislación vigente obliga a las comunidades autónomas a desarrollar programas y recursos que propicien, más allá de la separación familiar, otro tipo de medidas de preservación individualizadas, preventivas y positivas, encaminadas no sólo a eliminar los factores de riesgo, sino también a potenciar los de protección, dejando atrás el carácter puramente clínico o asistencial típicamente dirigido a las situaciones de desamparo (De Paul, 2009; Rodrigo et al., 2008).

Dado que este artículo se refiere a un trabajo que se ha realizado en Andalucía, a continuación se va a describir, brevemente, cómo se organiza la intervención familiar en los Servicios Sociales de esta comunidad autónoma. Estos dispositivos se rigen por la Ley 2/1988 del 4 abril, la cual tiene por objeto:

Regular y garantizar en el ámbito de la Comunidad Autónoma Andaluza un Sistema Público de Servicios Sociales que ponga a disposición de las personas y de los grupos en que ésta se integran recursos, acciones y prestaciones para el logro de su pleno desarrollo, así como la prevención, tratamiento y eliminación de las causas que conducen a su marginación (art. 1).

\section{Este sistema comprende:}

Aquellos recursos, actividades y prestaciones organizadas para la promoción del desarrollo de los individuos y grupos sociales para la obtención de mayor bienestar personal y una 
mejor calidad de vida, así como para la prevención y eliminación de la marginación social (art. 4).

Y se inspira, según el artículo 2, en los principios de responsabilidad pública, solidaridad, igualdad y universalidad, participación, prevención, globalidad, normalización e integración, planificación y coordinación, y finalmente, descentralización. En el artículo 5, los Servicios Sociales se estructuran en dos niveles de intervención: Servicios Sociales comunitarios y Servicios Sociales especializados. Así como los primeros se dirigen a familias en riesgo, los segundos atienden situaciones de desamparo que requieren una especial protección social, por medio de recursos o programas específicos. Dado que los resultados que se presentan en este artículo tienen que ver con familias usuarias de la primera de las modalidades de intervención, a continuación se detallan cuáles son las prestaciones disponibles desde este nivel de atención en Andalucía.

Los Servicios Sociales comunitarios constituyen la estructura básica del sistema público de Servicios Sociales de Andalucía, y su responsabilidad recae en las corporaciones locales. Desde este nivel de atención, se ofrecen una serie de prestaciones concretas que quedan recogidas en el artículo 10 de la Ley 2/1988, y que se han materializado en una estructura en atención a familias con prestaciones dirigidas a toda la población, entre las que hay que destacar los siguientes servicios:

1. Servicio de información y orientación. Sus actuaciones están encaminadas a informar a los ciudadanos sobre sus derechos y los recursos sociales existentes, y a detectar y analizar los problemas de los distintos sectores de la población. Se considera la puerta de acceso a los demás servicios y prestaciones del sistema.

2. Servicio de convivencia y reinserción social. Sus acciones están dirigidas al apoyo profesional a personas y familias en las que se detectan problemas de relación entre sus miembros o bien en las relaciones que éstos mantienen con su entorno social, procurando su integración en la comunidad.

3. Programa de tratamiento familiar: Se desarrolla mediante convenios con las corpora- ciones locales. Las familias con las que trabajan estos equipos son derivadas de otros dispositivos de los Servicios Sociales o de la propia Delegación Provincial para la Igualdad y Bienestar Social, cuyos profesionales habrán valorado ya, tras una intervención previa, que se requiere un tratamiento específico para obtener cambios significativos que eviten la separación familiar. Este programa supone el último recurso disponible para las familias, en una lógica de intervención de menor a mayor intensidad, antes de una medida de amparo.

En definitiva, el panorama actual de la intervención familiar en España es notablemente distinto al de hace unas décadas: hemos pasado de un enfoque asistencialista, centralizado, y que se ocupaba casi exclusivamente de las situaciones más graves (con medidas basadas en la separación del menor y su familia), a otro en el que el sistema de protección se conceptúa como un derecho social básico de los ciudadanos, sus competencias se descentralizan y diversifican, y se amplían las realidades a las que dar cobertura y los enfoques de intervención con las mismas.

Como ya hemos avanzado, estos cambios han propiciado que, en nuestro país, se hayan desarrollado diversas investigaciones sobre familias usuarias de dispositivos de protección social. En el caso específico de las que se encuentran en riesgo psicosocial y reciben intervenciones de preservación y fortalecimiento familiar, hay que destacar los estudios mencionados de Rodrigo de la Universidad de la Laguna (Rodrigo et al., 2008; Rodríguez et al., 2006) y los trabajos de Hidalgo de la Universidad de Sevilla (Hidalgo, Lorence, et al., 2009; Menéndez, Hidalgo, Jiménez, Lorence y Sánchez, 2010). Igualmente son destacables los informes de distintas entidades u organismos públicos o privados interesados en el estudio de familias en situación de riesgo o exclusión social (Dirección General de Inclusión Social, 2004; Del Río, 2008; Instituto Nacional de Estadística, 2009) o de los propios profesionales del Servicio (Garrido y Grimaldi, 2009).

Este artículo se enmarca en una investigación más amplia, desarrollada en colaboración con el Área de Bienestar Social de la Diputación de Huelva, en la que hemos analizado algunas de las principales características indivi- 
duales, interpersonales, familiares y sociales de una muestra de familias en situación de riesgo que reciben intervenciones de preservación familiar desde los Servicios Sociales comunitarios. En concreto, se exponen algunos de los resultados obtenidos y los análisis efectuados con objeto de realizar una caracterización sociodemográfica de estas familias, de sus condiciones laborales y económicas, así como de su trayectoria y evolución en los Servicios Sociales.

Más allá de la frecuente tendencia a destacar los rasgos más negativos de estas situaciones, nos proponemos examinar tanto las debilidades como las fortalezas que presentan estas familias en los ámbitos evaluados, y por tanto delimitar, respecto a los mismos, tanto sus necesidades como sus recursos de cara a las intervenciones que con ellas se llevan a cabo, dado que es preciso tener en cuenta ambas cuestiones para diseñar medidas efectivas (Hidalgo, Menéndez et al., 2009; Rodrigo et al., 2008).

\section{Método}

\subsection{Participantes}

La muestra está formada por 67 familias usuarias de los Servicios Sociales comunitarios de la Diputación de Huelva por razones de preservación familiar sobre las que se está interviniendo desde el Servicio de Atención Familiar y los Equipos de Tratamiento Familiar. Las responsabilidades del segundo ya han sido descritas en la introducción de este artículo, y las funciones de los Servicio de Atención Familiar se corresponden a las ya comentadas para el Servicio de convivencia y reinserción social. Las participantes se distribuyen de forma muy similar en estos dos dispositivos: un 49,3 por ciento son usuarias del Servicio de Atención Familiar y un 50,7 por ciento de los Equipos de Tratamiento Familiar.

\subsection{Instrumentos}

Los resultados que se describen en este trabajo tienen relación con diversos indicadores sociodemográficos y con la trayectoria en los Servicios Sociales de estas familias. Para evaluarlos se utilizó la entrevista de perfil sociodemográfico (Hidalgo, Menéndez, López, Sánchez, Lorence y Jiménez, 2006). Se trata de una entre- vista semiestructurada que permite recabar información de diversa índole sobre las características individuales de los componentes de la familia, así como los indicadores socioeconómicos que caracterizan estos hogares. La entrevista de perfil sociodemográfico incluye asimismo un espacio destinado a elaborar el genograma familiar e indicar algunos datos respecto a la estabilidad y la composición de la familia. Finalmente, la entrevista recopila información sobre el historial y la situación actual del adulto y su familia en relación con los Servicios Sociales, al tiempo que solicita la valoración que el profesional hace, mediante una escala de 0 a 10, de la evolución del usuario en la intervención.

\subsection{Procedimiento}

Para este estudio se ha contado con la colaboración de 12 profesionales de los Servicios de Atención Familiar y de los Equipos de Tratamiento Familiar de la provincia de Huelva. El equipo de investigación mantuvo una reunión con estos profesionales, en la que se les detallaron los objetivos del estudio, las características de la población diana, y el procedimiento para recabar los datos. Posteriormente se realizaron contactos personalizados con cada profesional para concretar los detalles de su participación en el estudio. Tras estos segundos contactos, cada profesional seleccionó a un grupo de usuarias y, después de recabar su colaboración en el estudio, completó el instrumento descrito anteriormente.

\section{Resultados}

A continuación se exponen los resultados obtenidos, a propósito de indicadores referidos al perfil sociodemográfico de las familias participantes en este estudio, su situación laboral y el nivel de pobreza y, por último, su trayectoria en los Servicios Sociales. Al hilo de esta exposición, se irán ofreciendo algunos comentarios sobre el significado y la interpretación que, en nuestra opinión, cabe hacer sobre los datos obtenidos. Los resultados y argumentos que se van a exponer se retoman, de manera conjunta, en la discusión final de este trabajo, con algunas de las conclusiones que, a nuestro juicio, se pueden extraer del mismo, en cuanto a las debilidades y las fortalezas que presentan estas familias en los ámbitos analizados. 


\subsection{Perfil sociodemográfico}

Las familias que han participado en esta investigación estaban integradas por aproximadamente cuatro personas $(M=3.98, D T=1.30)$, y tenían una media de dos hijos o hijas $(M=2.28, D T=1.08)$, de los cuales en torno dos $(M=1.78, D T=0.69)$ eran menores de edad. El 23,1 por ciento de estas familias convive con otras personas, fundamentalmente miembros de la familia extensa (sobre todo los abuelos). Se trata de núcleos familiares con una composición mayoritariamente estable $(84,6$ por ciento) y entre los que destacan las familias bajo responsabilidad exclusiva de mujeres: el porcentaje de hogares monomarentales se sitúa en un 41,8 por ciento $y$, de los biparentales (un 58,2 por ciento de la muestra), un 35,89 por ciento son familias reconstituidas.

En líneas generales, estos datos concuerdan con los resultados encontrados en otros trabajos interesados en caracterizar a familias en riesgo que se han llevado a cabo en España. Mención especial merece el elevado porcentaje de familias a cargo de madres solas, que supera muy ampliamente el 10 por ciento que caracteriza al total de familias españolas con menores de edad (Flaquer, Almena y Navarro, 2006). Este resultado es coherente con la constatada sobrerrepresentación de las familias monomarentales en los colectivos socialmente excluidos o con un elevado riesgo de estarlo ${ }^{1} \mathrm{y}$ con los estudios específicamente realizados con familias usuarias de los Servicios Sociales por razones de preservación familiar (Hidalgo, Menéndez et al., 2009; Martín, Máiquez, Rodrigo, Correa y Rodríguez, 2004; Menéndez et al., 2010; Rodrigo, Correa, Maíquez, Martín y Rodríguez, 2006; Rodríguez et al., 2006). Aunque en este artículo no se han efectuado análisis específicos para caracterizar el perfil psicosocial de estas familias, hay que señalar el modesto papel que en ellas juega el padre de los hijos desde el punto de vista económico, dado que sólo en un 36,4 por ciento de los casos contribuye con una pensión al sustento familiar.

En lo que respecta a las viviendas de estas familias, su tamaño medio aproximado es de $96 \mathrm{~m}^{2}(M=96.20, D T=43.71)$, en un rango entre $30 \mathrm{~m}^{2}$ y $245 \mathrm{~m}^{2}$. Hay que destacar que la correlación entre el tamaño del hogar y el número de personas que en él residen no es significativa $(r=-.019, p=.882)$, es decir, las familias más numerosas no necesariamente viven en hogares más amplios. Además, al dividir el tamaño de la vivienda entre el número de residentes, los resultados indican que el 21,9 por ciento de las familias viven en hogares con menos de $15 \mathrm{~m}^{2}$ por persona, nivel a partir del cual puede hablarse de unas condiciones de hacinamiento severo según los estándares poblacionales (Laparra y Pérez, 2009). De nuevo estos datos son coherentes con los resultados de otros trabajos (Menéndez et al., 2010), los cuales también ponen de manifiesto que la amplitud de la vivienda y el número de personas que en ella viven no están relacionados entre sí. En síntesis, los datos respecto a la situación residencial indican que muchas de las familias viven en hogares pequeños y saturados, con un nivel objetivo de hacinamiento que afecta a algo más de la quinta parte de la muestra.

Los análisis efectuados, en función del tipo de servicio que desarrolla intervenciones de preservación familiar, muestran una variabilidad en los indicadores anteriores. Los resultados arrojan que, en comparación con las familias del Servicio de Atención Familiar, las de los Equipos de Tratamiento Familiar son más numerosas $(F=5.12, p=.027)$ y residen en hogares más pequeños $(F=4.38, p=.040)$ en los que, además, existe un índice mayor de hacinamiento $\left(\chi^{2}=4.66, p=.030\right)$.

\subsection{Situación económica y nivel de pobreza}

Los análisis realizados para examinar la situación económica de estas familias muestran que cuentan con unos ingresos mensuales medios aproximados de 945.44 euros $(D T=604.92)$, que son inestables en un 45,3 por ciento de las ocasiones. Con respecto a la procedencia de los mismos, los resultados revelan una notable dependencia económica en estas familias: el 62 por ciento de ellas reciben ayudas sociales, que suponen su única fuente de ingresos en un 14 por ciento de los casos.

Más allá de los resultados sobre la cuantía y la procedencia de los ingresos familiares, se efectuaron distintos cálculos adicionales para

\footnotetext{
${ }^{1}$ Para un análisis de esta circunstancia en España, véase Flaquer et al., 2006; Laparra y Pérez, 2009.
} 
aportar significado a los mismos. Para ello se tomó en consideración el número de personas que vivían con dichos ingresos, en comparación con estándares poblacionales. El resultado de esta comparación refleja, por tanto, en qué medida una familia determinada dispone de unos ingresos anuales ponderados por unidad de consumo que están por encima o por debajo del umbral poblacional de pobreza. Así, los niveles oficiales de pobreza en Andalucía (INE, 2009) son pobreza moderada (60 por ciento de la mediana), para ingresos familiares entre 5.528 euros y 3.686 euros; pobreza alta (40 por ciento de la mediana), para ingresos entre 3.685 euros y 2.671 euros; y pobreza severa ( 25 por ciento de la mediana), para ingresos inferiores a 2.670 euros. Los análisis realizados muestran que la mayoría de estas familias (el 59,6 por ciento) cuentan con unos recursos económicos que estaban por debajo del límite a partir del cual puede hablarse, objetivamente, de una situación económica no ya precaria sino de pobreza. En concreto, los ingresos medios ponderados se sitúan en 203.45 euros $(D T=$ 3.378.70), por debajo del umbral de pobreza de acuerdo con los datos autonómicos.

La Figura 1 presenta un análisis más detallado de estos resultados, diferenciando las familias en situación de pobreza más o menos severa según los criterios estandarizados que se acaban de especificar. Como puede apreciarse, el grupo de familias pobres es el más numeroso, el que se caracteriza por condiciones económicas más severas: el 25 por ciento de la muestra vive con unos ingresos anuales ponderados muy reducidos $(M=2.182 .79$ ?, $D T=438.15)$. Los resultados obtenidos difieren de los datos nacionales para la población general. Así, mientras que en España un 19,6 por ciento de la población general se encuentra por debajo del umbral de pobreza (INE, 2009), en este estudio el porcentaje de familias pobres asciende al 60 por ciento.

Como puede apreciarse, y coincidiendo con otras investigaciones con población usuaria de los Servicios Sociales (Dirección General de Inclusión Social, 2004; Garrido y Grimaldi, 2009), los resultados descritos señalan la considerable precariedad económica y laboral en la que viven la mayor parte de estas familias. Teniendo en cuenta los indicadores encontrados (ingresos reducidos, inestables en algo me-

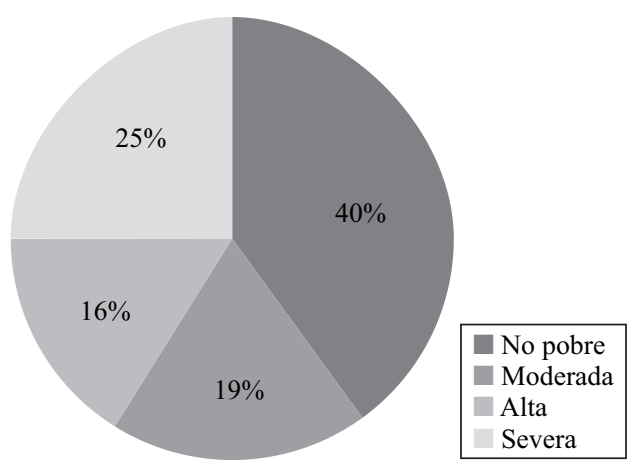

Figura 1. Distribución de las familias en función del nivel de pobreza.

nos de la mitad de las ocasiones, y que están por debajo del umbral de la pobreza en el 60 por ciento de los casos), no resulta sorprendente que más de la mitad (el 62 por ciento) de las familias sean receptoras de ayudas sociales, circunstancia ésta que, aunque sin duda contribuye a aliviar su difícil situación económica, también propicia la dependencia de ayudas externas a la que ya hemos hecho referencia.

La comparación de los indicadores anteriores en función del tipo de servicio revela algunas diferencias estadísticamente significativas. Así, las familias cuyos ingresos provienen exclusivamente de alguno de sus miembros son fundamentalmente usuarias del Servicio de Atención Familiar, mientras que la mayoría de las que combinan el trabajo con ayudas sociales reciben intervenciones de los Equipos de Tratamiento Familiar $\left(\chi^{2}=6.73, p=.035\right)$. Por otro lado, las familias con las que se trabaja el Servicio de Atención Familiar tienen unos ingresos ponderados que se sitúan por encima del umbral de pobreza, mientras que los recursos económicos de las tratadas por los Equipos de Tratamiento Familiar son inferiores a dicho límite $(F=3.02, p=.089)$.

\subsection{Historial y situación actual en los Ser- vicios Sociales}

En cuanto a la relación con los Servicios Sociales, los resultados obtenidos reflejan una importante variabilidad en el número de años que la familia llevaba siendo usuaria de algún servicio. Así, y aunque la media se sitúa aproximadamente en tres años $(M=2.94$, 


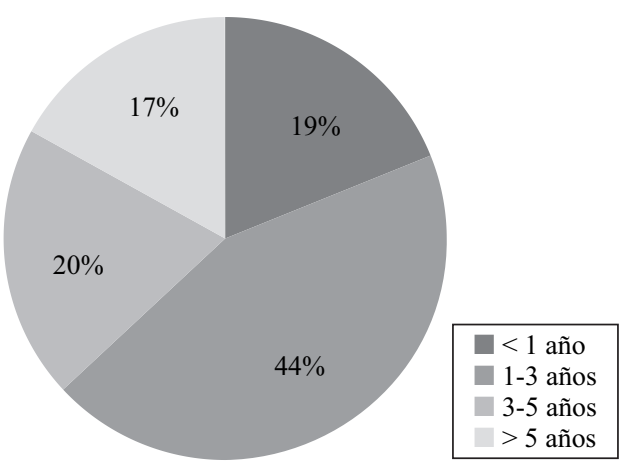

Figura 2. Distribución de las familias en función de la trayectoria en los Servicios Sociales.

$D T=3.62)$, los valores mínimo y máximo de la distribución muestral oscilan desde familias con ocho meses hasta una con 22 años. En la Figura 2, aparece representada esta distribución mediante categorías.

Estos resultados difieren de los datos poblacionales andaluces. Así, en el estudio de Garrido y Grimaldi (2009) el 36,8 por ciento de las familias que están siendo intervenidas por los Servicios Sociales en Andalucía mantienen un expediente activo de 5 o más años de antigüedad, mientras que en esta investigación sólo se encontró el 17,2 por ciento.

Además del Servicio de Atención Familiar o los Equipos de Tratamiento Familiar, muchas de las familias de este estudio son también usuarias de otro tipo de servicios, tanto ofrecidos por los Servicios Sociales como por otros dispositivos de bienestar social. Como puede apreciarse en la Tabla 1, en el 18,5 por ciento de los casos las intervenciones desarrolladas por el Servicio de Atención Familiar o por los Equipos de Tratamiento Familiar se combinaron con las ofrecidas desde los Servicios de Información y Orientación, pero los porcentajes para el resto de las prestaciones son reducidos. Sin embargo, las familias de la muestra suelen utilizar, en mayor medida, prestaciones de otros dispositivos de bienestar social, especialmente las que ofrecen: Salud Mental, los Centros de la Mujer y los relacionados con el fomento del empleo. Estos datos indican que la coordinación entre los servicios de atención y apoyo a las familias, tanto de bienestar social como de otros dispositivos, es un punto funda-

\begin{tabular}{|l|r|}
\hline \multicolumn{2}{|l|}{ Servicios sociales } \\
\hline Servicio de Información y Orientación & $18.5 \%$ \\
\hline Ayuda económica familiar & $7.7 \%$ \\
\hline Servicio de Ayuda Domiciliaria & $4.6 \%$ \\
\hline Ayuda de emergencia social & $3.1 \%$ \\
\hline Programa Comprende y Educa & $1.5 \%$ \\
\hline $\begin{array}{l}\text { Talleres de Convivencia e Inserción } \\
\text { Social }\end{array}$ & $4.6 \%$ \\
\hline $\begin{array}{l}\text { Programas de prevención para adoles- } \\
\text { centes }\end{array}$ & $1.5 \%$ \\
\hline $\begin{array}{l}\text { Programas de prevención, seguimiento } \\
\text { y control del absentismo }\end{array}$ & $1.5 \%$ \\
\hline Otros dispositivos de bienestar social \\
\hline Salud Mental & $49.2 \%$ \\
\hline Centro Comarcal de Drogodependencias & $10.8 \%$ \\
\hline Centros de la Mujer & $29.2 \%$ \\
\hline Empleo & $27.7 \%$ \\
\hline Vivienda & $21.2 \%$ \\
\hline Otros & $26.9 \%$ \\
\hline
\end{tabular}

Tabla 1. Prestaciones de las que son usuarias las familias de la muestra.

mental para realizar intervenciones integradas y apropiadas con las mismas.

Se solicitó a los profesionales que trabajaban con estas familias que describieran, mediante una escala de 0 a 10 , la evolución más o menos positiva que, en su opinión, caracterizaba a las familias del estudio. Los resultados se sitúan alrededor de una media de 6.31 $(D T=1.56)$, con un mínimo de 3 (el 4,7 por ciento de las familias) y un máximo de 10 (1,6 por ciento de los casos). En concreto, las familias usuarias del Servicio de Atención Familiar presentan una evolución que alcanza una media de $6.70(D T=1.34)$, mientras que las de los Equipos de Tratamiento Familiar es de $5.97(D T=1.68)$. Los análisis efectuados señalan algunas diferencias estadísticamente relevantes en los indicadores anteriores en función del tipo de servicio. Así, y aunque con una significación marginal, los análisis de varianza indican que las familias que reciben in- 
tervenciones de los Equipos de Tratamiento Familiar tienden a tener una trayectoria más prolongada en los Servicios Sociales $(F=3.44, p=.068)$, a ser usuarias de un mayor número de prestaciones $(F=3.57$, $p=.063)$, y a tener una evolución menos positiva, según los profesionales $(F=3.62$, $p=.062$ ). Así, pues, atendiendo a los datos que facilitaron los técnicos de Servicios Sociales, se encuentra un perfil de mayor precariedad y dificultad entre las familias usuarias de los Equipos de Tratamiento Familiar.

No disponemos de datos nacionales al respecto pero Garrido y Grimaldi (2009), en su estudio con 770 familias usuarias andaluzas, han examinado la relación entre el nivel de riesgo y el servicio que trabaja con la familia. En consonancia con los resultados presentados en este artículo, su investigación mostró que la severidad del riesgo de las familias tendía a ser acorde con el nivel de intervención recibida, en concreto, las familias atendidas por los Servicios de Convivencia y Reinserción Social (similar al Servicio de Atención Familiar en la Diputación de Huelva) suelen presentar un índice de riesgo ligeramente inferior a las familias atendidas por los Equipos de Tratamiento Familiar.

\section{Conclusiones}

Los resultados presentados en este trabajo indican, en primer lugar, que las familias en situación de riesgo usuarias de los Servicios Sociales por razones de preservación familiar tienen un perfil sociodemográfico muy variable y diverso que, en términos generales, está caracterizado por algunos rasgos positivos y muchos negativos. Así, y al igual que en otras investigaciones con este tipo de familias, las que han participado en este estudio ofrecen un perfil que, salvo excepciones, resulta muy heterogéneo. Sin embargo, más allá de esta variabilidad, existen algunas características muy frecuentes en estas familias que tienden a definir una realidad fuertemente marcada por la adversidad y la precariedad a diversos niveles.

Las familias de este estudio tienen tamaño moderado y muy fundamentalmente son estables en cuanto a su composición, pero, también en muchas ocasiones, tratan de funcionar como contextos de desarrollo y educación para los menores en condiciones de precariedad re- sidencial y sobre todo económica. Muchas de las familias de la muestra viven en hogares pequeños, saturados, y que en uno de cada cinco casos ofrecen condiciones objetivas de hacinamiento; asimismo, sus ingresos son reducidos, inestables en algo menos de la mitad de los casos, y propician, en el 60 por ciento de las ocasiones, que estas familias vivan por debajo del nivel de pobreza y que acaben dependiendo de ayudas sociales.

En este trabajo no se han efectuado análisis específicos al respecto, pero en otro estudio que hemos llevado a cabo con familias sevillanas en riesgo también hemos constatado la sobrerrepresentación de los hogares monomarentales que ha aparecido en esta muestra, y hemos podido comprobar que las condiciones de precariedad económica y laboral son particularmente elevadas en las familias a cargo de madres solas.

En segundo lugar, el historial y la situación actual de estas familias en los Servicios Sociales también están caracterizados por la variabilidad pero, al mismo tiempo, por algunas características bastante frecuentes. Así, algo menos de la mitad de las familias tiene una trayectoria moderada en los Servicios Sociales (entre uno y tres años), resultado que es coherente con el nivel específico de riesgo que caracteriza a los servicios de preservación familiar, en los que, como es conocido, si el caso no responde positivamente a la intervención, tras dos o tres años, debe ser derivado a otro servicio más especializado, al tiempo que deben retornar a este nivel las familias con circunstancias más complejas y problemáticas que han respondido positivamente a intervenciones de más calado.

Con toda probabilidad, el (reducido) porcentaje de casos con una trayectoria más prolongada se corresponde con esta última circunstancia. Por otra parte, y de nuevo en consonancia con el nivel de riesgo de la muestra (recuérdese que no incluye medidas de desamparo), en opinión de los profesionales que trabajan con estas familias su evolución es mayoritariamente positiva. El importante porcentaje de familias que combinan las intervenciones de preservación familiar con otras prestaciones, especialmente las ofrecidas desde los Servicios de Salud Mental y los Centros de la Mujer, no hace sino constatar la depen- 
dencia de ayudas y apoyos externos que existe en estos casos.

La tercera conclusión que se extrae de los resultados presentados tiene que ver con las diferencias entre los dos servicios, que ponen de manifiesto un perfil de mayor adversidad entre las familias de los Equipos de Tratamiento Familiar. Como hemos descrito, éstas viven en peores condiciones residenciales, presentan un nivel de pobreza más elevado y dependen en mayor medida de ayudas económicas, al tiempo que tienen, en los Servicios Sociales, una trayectoria más prolongada, una evolución menos positiva, y una mayor dependencia de apoyos externos, pues utilizan más otras prestaciones. Esta mayor precariedad, tanto residencial y económica como en la relación con los servicios de protección social, es coherente con el mayor nivel de riesgo que suele caracterizar a la población usuaria de los Equipos de Tratamiento Familiar, y avala en nuestra opinión la pertinencia de las intervenciones que se llevan a cabo desde este servicio, habitualmente de más intensidad que las desarrolladas en el Servicio de Atención Familiar.

En definitiva, creemos que los resultados presentados en este artículo indican que las familias en situación de riesgo psicosocial tienen, en los niveles explorados, necesidades de intervención pero también recursos y fortalezas como contextos de desarrollo que deben ser tomadas en consideración en el diseño de las medidas que con ellas se llevan a cabo. Sin duda sus necesidades vienen definidas por la precariedad (económica, residencial, y en la relación con los servicios de protección social) y la dependencia que de ésta se deriva, pero los profesionales que trabajan con estas familias también deben, en nuestra opinión, tener presente que se trata de familias mayoritariamente estables y que responden positivamente a la intervención.

\section{Referencias bibliográficas}

Consejo de Europa (2006). Recommendation 19 of the Committee of Ministers to member states on policy to support positive parenting. Explanatory Report. Estrasburgo: Consejo de Europa.

De Paul, Joaquín (2009). La intervención psicosocial en protección infantil en España: evolución y perspectivas. Papeles del Psicólogo 30, 4-12.

Del Río, Rafael (coord.) (2008). IV Informe sobre exclusión y desarrollo social en España. Madrid: Fundación FOESSA.

Dirección General de Inclusión Social (2004). Sistema de Información de Usuarios de Servicios Sociales (SIUSS). Datos generales 2004. Recuperado de: www.mtas.es/SGAS/ServiciosSocDep/ServiciosSociales/siuss/SIUSS.htm.

Flaquer, Lluis, Almeda, Elisabeth y Navarro, Lara (2006). Monoparentalidad e infancia. Barcelona: Fundación «La Caixa».

Garrido, Miguel, y Grimaldi, Víctor (2009). Evaluación del Riesgo Psicosocial en Familias Usuarias del Servicio Público de Servicios Sociales de Andalucía. Sevilla: Consejería para la Igualdad y Bienestar Social.

Hidalgo, Victoria, Lorence, Bárbara, Pérez, Javier, Menéndez, Susana., Sánchez, José, Jiménez, Lucía y Arenas, Ángela (2009). El apoyo social de mujeres solas con responsabilidad familiar. Un estudio con madres de los Servicios Sociales Comunitarios. Sevilla: Instituto Andaluz de la Mujer.

Hidalgo, Victoria, Menéndez, Susana, López, Isabel, Sánchez, José, Lorence, Bárbara y Jiménez, Lucía (2006). Entrevista de Perfil Sociodemográfico (PSD). Documento inédito. Universidad de Sevilla, Sevilla.

Hidalgo, Victoria, Menéndez, Susana, Sánchez, José, Lorence, Bárbara y Jiménez, Lucía (2009). La intervención con familias en situación de riesgo psicosocial. Aportaciones desde un enfoque psicoeducativo. Apuntes de Psicología 23, 413-426.

INE (2009). Estudio descriptivo de la pobreza en España. Recuperado del sitio Web del Instituto Nacional de Estadística. Recuperado de: www.ine.es.

Laparra, M. y Pérez, B. (2009). La exclusión social en España: un espacio diverso y disperso en intensa transformación. En VI Informe sobre exclusión y desarrollo social en España 2008 (pp. 175-297). Madrid: Fundación FOESSA. 
Ley 2/1988, de 4 de Abril, de los Servicios Sociales de Andalucía. BOJA núm. 29 del 12 de Abril de 1988.

Ley 1/1996, de 15 de Enero, de Protección Jurídica del Menor, de modificación parcial del Código Civil y de la Ley de Enjuiciamiento Civil. BOE núm. 15 del 17 de Enero de 1996.

López, Félix. (2008). Necesidades en la infancia y la adolescencia: respuesta familiar, escolar y social. Madrid: Pirámide.

Maíquez, $\mathrm{M}^{\mathrm{a}}$ Luisa y Capote, Carmen (2001). Modelos y enfoques en Intervención Familiar. Intervención Psicosocial 10 (2), 185-198.

Martín, Juan Carlos, Máiquez, $\mathrm{M}^{\mathrm{a}}$ Luisa, Rodrigo, $\mathrm{M}^{\mathrm{a}}$ José, Correa, Nieves y Rodríguez, Juan (2004). Evaluación del programa «Apoyo personal y familiar» para madres y padres en situación de riesgo psicosocial. Infancia y Aprendizaje, 27, 437-445. d.o.i.:10.1174/0210370042396887.

Menéndez, Susana, Hidalgo, Victoria, Jiménez, Lucía, Lorence, Bárbara y Sánchez, José (2010). Perfil psicosocial de familias en situación de riesgo. Un estudio de necesidades con usuarias de los Servicios Sociales Comunitarios por razones de preservación familiar. Anales de Psicología, 26, 378-389.

Palacios, Jesús, y Rodrigo, $\mathrm{M}^{\mathrm{a}}$ José. (1998). La familia como contexto de desarrollo humano. En M. J. Rodrigo y J. Palacios (coords.). Familia y desarrollo humano, 25-44. Madrid: Alianza Editorial.

Rodrigo, M. J., Correa A. D., Máiquez, M. L., Martín. C. y Rodríguez G. (2006). Family Preservation Services on the Canary Islands. European Psychologist, 11, 57-70. d.o.i.:10.1027/10169040.11.1.57.

Rodrigo, Ma José, Máiquez, $\mathrm{M}^{\mathrm{a}}$ Luisa, Martín, Juan Carlos, y Byrne, Sonia. (2008). Preservación familiar. Un enfoque positivo para la intervención con familias. Madrid: Ediciones Pirámide.

Rodríguez, Guacimara, Camacho, Juan, Rodrigo, Ma José, Martín, Juan Carlos y Máiquez, Ma Luisa (2006). Evaluación del riesgo psicosocial en familias usuarias de servicios sociales municipales. Psicothema, 18, 200-206.

Rodríguez, Iván y Menéndez, Susana (2003). El resto de las nuevas realidades familiares. Portularia: Revista de Trabajo Social, 3, 9-32. 